$$
\begin{gathered}
\frac{\mathrm{d} k}{\mathrm{~d} p}=\frac{4 k_{0}+4 p / 9}{k_{0}+p / 3}, \\
k=k_{0}+4 p / 3+8 k_{0} \log \left(1+p / 3 k_{0}\right) .
\end{gathered}
$$

With equations (4) and (5) and the assumed values for the earth's interior, we can now construct the accompanying table, where the units of $p$ and $k$ are $10^{12}$ dynes/cm.

\begin{tabular}{|c|c|c|c|}
\hline$p$ & $\mathrm{~d} k / \mathrm{d} p$ & $k$ & Depth \\
\hline 0 & 4 & 2 & 0 \\
1 & $3 \cdot 62$ & $5 \cdot 8$ & $2,200 \mathrm{~km}$. \\
2 & 3.33 & $9 \cdot 3$ & $3,500 \mathrm{~km}$. \\
3 & $3 \cdot 11$ & $12 \cdot 5$ & $4,800 \mathrm{~km}$. \\
4 & $2 \cdot 93$ & $15 \cdot 5$ & centre \\
\hline
\end{tabular}

In the construction of this table, the assumption of Bullen's compressibility pressure hypothesis is implicit, and his values giving the variation of pressure with depth have been used to estimate the approximate depth. It will be noticed that there is a very good correspondence between the values of $k$ obtained from equation (5) and Bullen's ${ }^{3}$ volues.

I wish to thank Prof. K. E. Bullen, of the University of Sydney, and Prof. G. Bosson, of the N.S.W. University of Technology, for their interest and assistance.

Wollongong Technical College,

\section{A. Keane}

Gladstone Avenue,

Wollongong, 5C,

New South Wales.

Feb. 23.

'Birch, F., J. Geophys. Res., 57, No. 12, 246 (1952). "Murnaghan, F. D., "Finite Deformation of an Elastic Solid", 70

'Bullen, K. E., "An Introduction to the Theory of Seismology", 220 (Camb. Univ. Press, 1947).

\section{An Extended Smith-Lagrange Invariant}

IT is well known in optics that, if $\mu_{r}$ is the refractive index of the $r$ th medium of a co-axial system, $h_{r}$ is the height of the image of a given object from the axis in the same medium, and $\alpha_{r}$ is the angle a selected ray makes with the axis at the image plane, then

$$
\mu_{r} h_{r}\left\{\begin{array}{l}
\sin \alpha_{r} \\
\tan \alpha_{r}
\end{array}\right.
$$

is invariant (Smith-Lagrange invariant). The choice of $\sin \alpha_{r}$ or $\tan \alpha_{r}$ varies with different authors. The relation holds in all systems where $\sin \alpha$ and $\tan \alpha$ are indistinguishable.

The development of various two-stage processes of image formation ${ }^{1}$, in which the wave-length may be altered between object and image, makes it profitable to consider whether the wave-length can be introduced. Since wave-length is inversely proportional to refractive index, the form :

$$
\frac{\mu_{r} h_{r} \sin \alpha}{\lambda_{r}}
$$

as invariant suggests itself. It is readily verified in the case of diffraction microscopy that this is correct.

Hopkins $^{2}$ has recently published a coherence criterion for a beam of circular cross-section :

$$
\frac{\mu h \sin \alpha}{\lambda}=\frac{z}{2 \pi} \ngtr 0 \cdot 16,
$$

in which $h$ and $\sin \alpha$ are capable of two alternative interpretations, one of which agrees with that of (1) and (2). Comparison with (2) shows at once that, if a beam is coherent in one image plane of a Gaussian co-axial system, it will be coherent in all other image planes. Thus coherence is conserved in such systems. Moreover, if the invariant (2) exceeds $0 \cdot 16$ in numerical magnitude, the degree of partial coherence has been shown by Hopkins to be $2 J_{1}(z) / z$, and is still invariant down the system, though now imperfect.

The Smith-Lagrange invariant thus corresponds to the physical law that the degree of coherence is conserved from image plane to image plane in Gaussian systems.

Department of Physics,

G. L. Rogers

\section{Victoria University College, Box 196,}

Wellington, N.Z.

${ }^{1}$ Boersch, H., Z. Tech. Phys., 19, 337 (1938). Bragg, W. I.., Nature, 143, 678 (1939) ; 149, 470 (1942). Buerger, M. J., Proc. U.S. Nat. Acad. Sci., 25, 383 (1939); 87, 117 (1941); 36, 330 (1950); J. App. Phys., 21, 990 (1950). Lipson, H., et al., Acta Cryst.; 777 (1948) ; Proc. Roy. Soc. A, 197, 454 (1949); Proc. Phys. Soc., B, 64, 449 (1951). Haine, M. E., and Mulvey, T., J. Opt. Soc. Amer., [42, 763 (1952)]. Haine, M. E., and Dyson, J., Nature, 166, $315(1950)$. Bragg, W. L., Nature, 166, 399 (1950); 167, 190 (1951). Rogers, G. L., Proc. Roy. Soc. Edin., 63, 193, [313] (1952).

${ }^{2}$ Hopkins, H. H., Proc. Roy. Soc., 208, 263 (1951).

\section{Isoxanthopterin}

WE have recently prepared isoxanthopterin (2amino-4 : 7-dihydroxypteridine) by a new synthesis, namely, the condensation of triamino-4-hydroxypyrimidine with ethyl glyoxylate hemiacetal. The synthesis, purification and analysis will be reported in detail elsewhere. The ultra-violet spectrum of the product consisted of three peaks at the $\lambda_{\max }$. values previously reported ${ }^{1,2}$, but the extinction coefficients were so much higher as to suggest that the samples previously examined contained no more than 80 per cent of isoxanthopterin. Our results are as follows (material ḋried at $150^{\circ}$, analysing as anhydrous, dissolved in $0 \cdot 1 \mathrm{~N}$ potassium hydroxide).

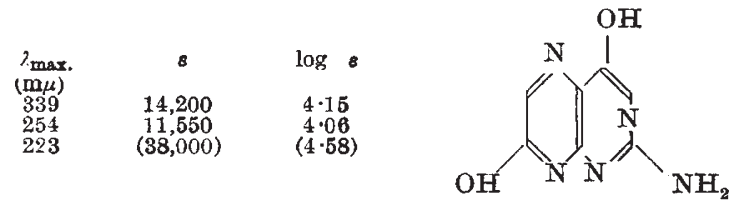

The $\varepsilon$ at $223 \mathrm{~m} \mu$ may be subject to a small error from stray light.

We wish to thank Dr. E. R. Holiday, of the Medical Research Council Spectrographic Research Unit, London Hospital, and Dr. F. Korte, of the Biochemische Abteilung, Chemisches Staatsinstitut of the University of Hamburg, for confirming the above spectrographic results, using our synthetic material. Dr. Korte tells us that previous spectra have been obtained only from material of natural origin.

ADRIEN ALBERT

Department of Medical Chemistry,

H. C. S. Woon

Australian National University,

183 Euston Road,

London, N.W.1.

May 4.

1 Hitttel and Sprengling, Annalen, 554, 69 (1943).

${ }^{2}$ Tschesche and Korte, Ber., 84, 801 (1951). 